changes by increasing physical activity and limiting calories (1200 ckal), and patients from Group 1 were additionally treated with orlistat $120 \mathrm{mg} 3$ times a day. WOMAC and EQ-5D scores and anthropometric data were regularly obtained during the study; biochemistry panel (lipid profile, glucose, CRP), leptin and interleukin- 6 were assessed at baseline and at Mo 6 .

Results: Metabolic syndrome (MS) was diagnosed at baseline in 41 patients. Following orlistat therapy pts from Group 1 managed to reduce their body weight by $10,07 \%(p<0,05$; Fig 1$)$, and waist circumference (WC) by $7,5 \%(p<0,05)$, improving the clinical course of OA: total WOMAC score was reduced by $55 \%(p<0,05$; Fig 2), and EQ-5D quality of life was improved by $44 \%(p<0,05)$. Patients from Group 2 lost only $0,88 \%$ of their body weight versus the baseline, and reduced WC by $1 \%$, without changes in EQ-5D scores. MS lab parameters - lipid profile and glucose - did not change significantly after orlistat treatment in Group 1. Patients from Group 2 showed significant increase in total cholesterol $(p<0,05)$ after 6 months of dieting and exercising, revealing direct correlation between raised LDL and triglycerides levels and total WOMAC scores. CRP dropped by $23,74 \%(p>0,05)$ in Group 1. More pronounced decrease of CRP was observed in pts losing more than $10 \%$ of their body weight $(p=0,07)$. Statistically significant decrease in leptin and IL-6 levels was documented in pts with orlistat-induced significant weight loss $(p<0,05)$.

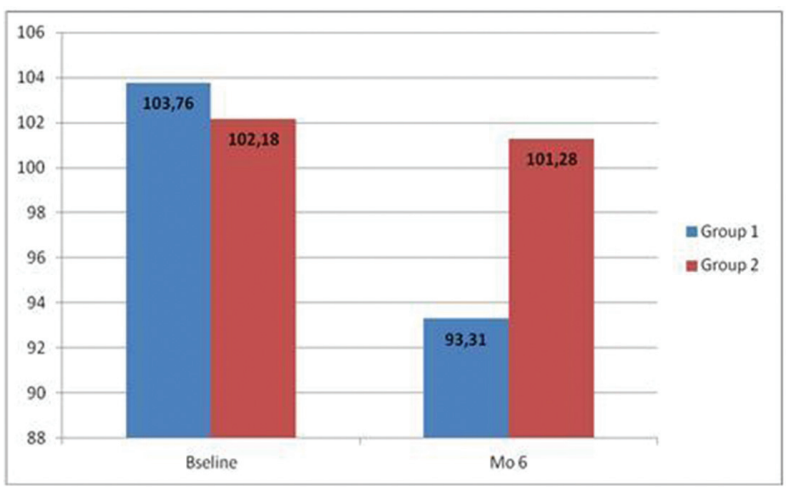

Abstract SAT0558 - Figure 1. Body mass reduction (kg, $p<0,05)$

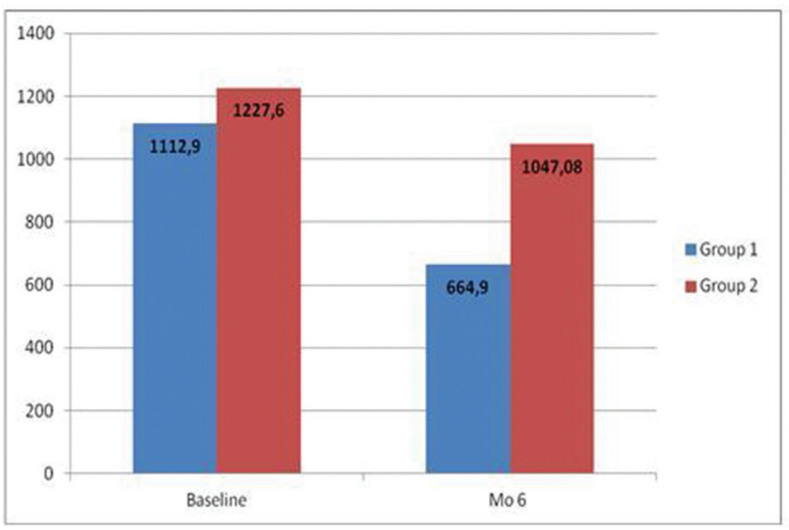

Abstract SAT0558 - Figure 2. Dynamics of total WOMAC score $(p<0,05)$.

Conclusions: Abdominal obesity is considered to be the major component of MS and key risk factor for the development and progression of knee OA. Our study demonstrates that induced by orlistat, diet and exercise weight loss by $>10 \%$ improves the clinical course of KOA and patient's quality of life, reduces activity of inflammation and improves key parameters of metabolic syndrome. Therefore, $\mathrm{KOA}$ and MS therapeutic strategies should include pharmacotherapy of obesity in patients who fail to lose weight with the diet and physical exercise only.

Disclosure of Interest: None declared

DOI: 10.1136/annrheumdis-2018-eular.5072

\section{SAT0559 SYSTEMATIC LITERATURE REVIEW (SLR) FOR THE 2018 UPDATE OF THE EULAR MANAGEMENT} RECOMMENDATIONS FOR HAND OSTEOARTHRITIS (OA)

F. Kroon ${ }^{1}$, M. Kloppenburg ${ }^{1,2}$, J. Schoones ${ }^{3}$, L. Carmona ${ }^{4} .{ }^{1}$ Rheumatology; ${ }^{2}$ Clinical Epidemiology, ${ }^{3}$ Walaeus Library, Leiden University Medical Center, Leiden, Netherlands; ${ }^{4}$ Institutode Salud Musculoesquelética, Madrid, Spain

Background: As part of the update of the EULAR management recommendations an SLR of available evidence for hand OA treatment was performed.

Objectives: To inform the task force of an overview of available evidence for the efficacy and safety of all non-pharmacological, pharmacological and surgical interventions for hand $\mathrm{OA}$.

Methods: We searched Medline, EMBASE, Cochrane Central, and 2016-2017 conference abstracts until June 2017 for (randomised) controlled trials or Cochrane reviews. Observational studies with a comparator were included to assess safety, or effects of surgical interventions. Main efficacy outcomes were pain, function, and hand strength. Risk of bias was assessed. Meta-analysis was performed if possible.

Results: Of 7036 records, 127 references were included, of which 50 studies concerned non-pharmacological, 64 concerned pharmacological, and 12 concerned surgical interventions. Most trials were published after 2007. Many studies were at high risk of bias, mainly due to inadequate randomisation or lack of blinding. Overall, effect sizes of effective therapies were small. Beneficial non-pharmacological treatments for pain relief include hand exercise, and prolonged splinting of the thumb base (Table). A single trial showed that assistive devices had no effect on pain, but led to functional improvements (mean difference (MD) -0.3 , $95 \%$ confidence interval $(\mathrm{Cl})-0.6 ; 0.01$ on AUSCAN function, range $1-5)$. Topical and oral non-steroidal anti-inflammatory drugs (NSAIDs) proved equally effective for pain relief (Table), while topical NSAIDs led to less adverse events (e.g., risk ratio for withdrawals due to adverse events $0.15,95 \% \mathrm{Cl} 0.03 ; 0.63$ ). Single trials demonstrated beneficial effects for other pharmacological interventions, including chondroitin sulphate, and intra-articular (i.a.) glucocorticoid injections in interphalangeal joints (Table). Compared to placebo, no beneficial effects were demonstrated for i.a. glucocorticoid or hyaluronic acid injections in the thumb base hydroxychloroquine, or tumour necrosis factor inhibitors (Table). In two trials, structure modification was the primary outcome measure, though no diseasemodifying properties were found in these studies. In a Cochrane review no surgical intervention for thumb base OA appeared more effective than another, although in general, more complex procedures led to more complications.

Abstract SAT0559 - Table 1. Effect on pain of main non-surgical interventions for hand OA from controlled trials.

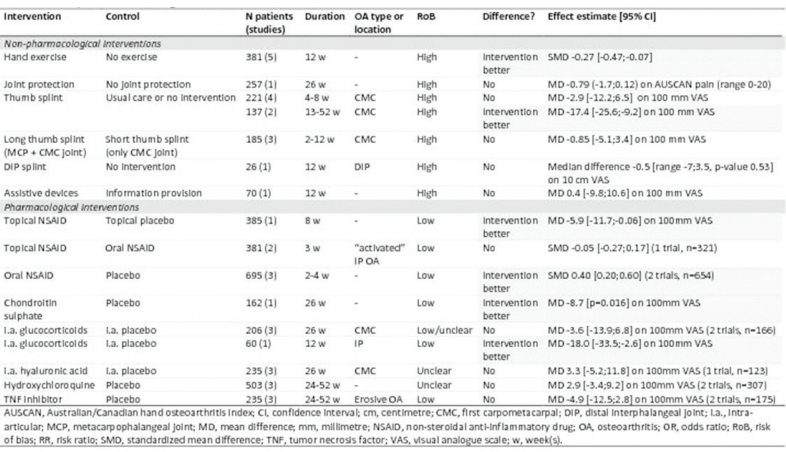

Conclusions: The number of trials in hand OA has steeply increased over the last decade, providing evidence for several therapeutic options for symptom relief, though no disease-modifying drugs have yet been found. High-quality trials investigating often used analgesics like paracetamol are needed.

Disclosure of Interest: None declared

DOI: 10.1136/annrheumdis-2018-eular.1963 